\title{
HPV vaccine decision making in pediatric primary care: a semi-structured interview study
}

\author{
Cayce C Hughes ${ }^{1}$, Amanda L Jones ${ }^{2}$, Kristen A Feemster ${ }^{2,3,4}$ and Alexander G Fiks ${ }^{1,2,4,5^{*}}$
}

\begin{abstract}
Background: Despite national recommendations, as of 2009 human papillomavirus (HPV) vaccination rates were low with $<30 \%$ of adolescent girls fully vaccinated. Research on barriers to vaccination has focused separately on parents, adolescents, or clinicians and not on the decision making process among all participants at the point of care. By incorporating three distinct perspectives, we sought to generate hypotheses to inform interventions to increase vaccine receipt.
\end{abstract}

Methods: Between March and June, 2010, we conducted qualitative interviews with 20 adolescent-mother-clinician triads (60 individual interviews) directly after a preventive visit with the initial HPV vaccine due. Interviews followed a guide based on published HPV literature, involved 9 practices, and continued until saturation of the primary themes was achieved. Purposive sampling balanced adolescent ages and practice type (urban resident teaching versus non-teaching). Using a modified grounded theory approach, we analyzed data with NVivo8 software both within and across triads to generate primary themes.

Results: The study population was comprised of 20 mothers (12 Black, $9<$ high school diploma), 20 adolescents (ten 11-12 years old), and 20 clinicians (16 female). Nine adolescents received the HPV vaccine at the visit, eight of whom were African American. Among the 11 not vaccinated, all either concurrently received or were already up-to-date on Tdap and MCV4. We did not observe systematic patterns of vaccine acceptance or refusal based on adolescent age or years of clinician experience. We identified 3 themes: (1) Parents delayed, rather than refused vaccination, and when they expressed reluctance, clinicians were hesitant to engage them in discussion. (2) Clinicians used one of two strategies to present the HPV vaccine, either presenting it as a routine vaccine with no additional information or presenting it as optional and highlighting risks and benefits. (3) Teens considered themselves passive participants in decision making, even when parents and clinicians reported including them in the process.

Conclusions: Programs to improve HPV vaccine delivery in primary care should focus on promoting effective parent-clinician communication. Research is needed to evaluate strategies to help clinicians engage reluctant parents and passive teens in discussion and measure the impact of distinct clinician decision making approaches on HPV vaccine delivery.

\section{Background}

Since 2006, vaccines have been licensed to prevent cervical cancer, genital cancers, precancerous lesions, genital warts, and other conditions caused by human papillomavirus (HPV) [1-3]. As a preventive intervention, the HPV vaccine is especially important since HPV is the most common sexually transmitted infection [4] and is often acquired shortly following sexual initiation [5]. The

\footnotetext{
* Correspondence: fiks@email.chop.edu

'Center for Pediatric Clinical Effectiveness and Policy Lab, The Children's Hospital of Philadelphia, 3535 Market Suite, Philadelphia, PA, 19104, USA Full list of author information is available at the end of the article
}

population at risk for acquiring HPV includes nearly half of all US high school students, [6] and an estimated $33.8 \%$ of 14 to 24 year olds are currently infected [4].

Multiple clinical trials have demonstrated the safety and efficacy of the HPV vaccine, $[7,8]$ and national guidelines recommend the HPV vaccine for girls 11-12 years of age as part of the routine adolescent vaccine platform [9]. However, despite these recommendations, rates of HPV vaccination remain the lowest of all adolescent vaccines according to the 2009 National Immunization Survey [10,11]. As of 2009 , only $27 \%$ of US girls between 13 and 17 years of age are fully vaccinated. Although the HPV

\section{Biomed Central}


vaccine is relatively new, this finding is especially concerning since full protection requires three doses given over a minimum of six months [9].

In an effort to increase vaccination rates, numerous studies have examined factors associated with the acceptability of HPV vaccine among parents and adolescents, and factors associated with clinician intention to vaccinate, both of which were high $[12,13]$. Importantly, parents, teens, and clinicians all report that their views on acceptability are influenced by each other. Effective communication about HPV among parents, teens and providers has been linked to vaccine acceptability and receipt $[14,15]$. Parents and adolescents are positively influenced by getting a doctor's recommendation,[16-18] adolescents stress the importance of parents, partners and other significant people supporting vaccination, especially mothers, [15,19-21] and clinicians' intention to vaccinate depends in part on their perception of parents' concerns regarding safety and efficacy [22-24]. Clinicians are also affected by normative influences such as national guidelines and recommendations $[24,25]$.

Prompted by the discrepancy between high reported vaccine acceptability and intention to vaccinate and low vaccination rates, we conducted a qualitative study using individual interviews with mother, adolescent, and primary care clinician triads to better understand decision making at the point of care. Discussion about barriers to vaccine receipt has generally focused separately on parents, clinicians or adolescents. We studied triads to move beyond conceptualizations of barriers among individuals, and to instead examine the interaction at the visit. By incorporating three distinct perspectives and focusing on decision making in the primary care setting, we sought to generate hypotheses to inform interventions to increase vaccine receipt.

\section{Methods}

This qualitative study was conducted within The Children's Hospital of Philadelphia (CHOP) Pediatric Research Consortium (PeRC), a multi-state, hospital-owned, primary care practice-based research network including more than 235,000 children and adolescents. Study practices included 4 urban teaching practices where fewer than 35\% of patients have private insurance and 5 practices not involved in resident teaching, where over $80 \%$ of children are privately insured. The study was conducted in a health system where the HPV vaccine was covered by insurance, which allowed us to look at factors other than cost that might impact the decision-making process.

\section{Study design and patient population}

We conducted semi-structured individual interviews with 20 mother-adolescent-clinician triads. Based on the emphasis in the existing literature on the impact of maternal input on HPV vaccine decision making, we excluded male caregivers $[14,15,18,26]$. We purposively sampled to achieve a balance of adolescent ages, (11-18) and a balance of practice settings, and conducted 60 interviews between March and June 2010. No more than three triads were chosen from each participating practice. We sampled only attending physicians, none of whom had specific training in adolescent medicine. Six triads declined; 5 due to parental or clinician time constraints and 1 due to an adolescent's medical complexity.

Rosters of eligible patients were generated from CHOP's electronic health record and included all girls ages 11-18 that had not initiated the HPV vaccine series and also had an upcoming well-visit at one of the participating sites. We chose to focus on preventive well-visits, as the American Academy of Pediatrics (AAP) recommends that immunizations be administered by primary care clinicians during these routine preventive visits and the HPV vaccine is typically discussed between US families and clinicians in this setting [27]. After a visit at which the adolescent had an opportunity to receive the HPV vaccine, we approached the mother, adolescent, and clinician to introduce the study and obtain consent/ assent. Interviews were conducted immediately after the visit, in a private room at the practice, and each member of the triad was interviewed separately. Interviews were conducted by either a pediatric resident (ALJ) or a nonclinical research assistant with experience in conducting qualitative interviews $(\mathrm{CCH})$. Themes were consistent across interviews. This study was approved by the CHOP Institutional Review Board, an official recognized research ethics committee.

\section{Data collection}

Through a detailed review of the relevant literature, meetings of the research team, and consultation with outside experts, we developed an interview guide to elicit parent, adolescent and clinician perspectives on the decision to recommend (clinician) and receive/refuse (mothers and adolescents) adolescent vaccines (guide available upon request). Parents and adolescents were asked about their prior knowledge of vaccines, whether they received vaccines at the visit, whether vaccines were discussed with the clinician, and how they made the final decision to either receive or refuse available vaccine(s). We asked clinicians to focus on the specific visit and describe the vaccine discussion, compare their presentation of HPV with other vaccines, and describe their perception of the family's decision. Two trained interviewers (AJS and $\mathrm{CCH}$ ) conducted the interviews. Themes were consistent across both interviewers. Using a brief questionnaire, we collected demographic data on participants. 


\section{Data analysis}

Interviews were audio taped and analyzed using NVivo qualitative data analysis software (QSR International, Melbourne, Australia). Analysis was based on a modified grounded theory approach, in which coding was inductive and not based on an a priori coding framework [28]. To take advantage of our study design, we analyzed data both within and across triads. Data collection was concurrent with data analysis, and continued until we reached saturation, with no new themes emerging. Two authors (ALJ and $\mathrm{CCH}$ ) independently coded the interviews. Using an iterative process, we reviewed codes, identified emerging themes, and resolved any discrepancies through consensus. Using NVivo, each transcript was linked with participants' demographic data. After all transcripts were coded, themes were finalized based on the consensus of the research team. Representative verbatim comments were selected for presentation.

\section{Results}

Nine adolescents received the HPV vaccine at the visit and 11 adolescents did not. All 11 that did not receive the HPV vaccine either received other routine adolescent vaccines at their visit or were already up to date, illustrating that HPV was the only vaccine refused. Demographic characteristics of participants are described in Table 1. We did not observe systematic patterns of vaccine acceptance or refusal based on adolescent age or years of clinician experience. Eight of the nine teens who accepted the $\mathrm{HPV}$ vaccine were African American. We identified three primary themes highlighted below. Verbatim comments to support these themes are presented in the text and in Table 2.

\section{Parents delayed, rather than refused vaccination, and when they expressed reluctance, clinicians were hesitant to engage them in discussion}

Only one mother in our sample was resolute in her decision never to vaccinate her daughter against HPV. Others who declined the vaccine at the visit planned to delay or "wait a year" and revisit the vaccine decision, as opposed to refusing the vaccine outright. One mother said, "We're going to wait on [Gardasil]...she's not even in high school yet...we're just going to wait and see...maybe when she's 16 or 17." When clinicians encountered hesitation from parents, they tended not to "push," instead affirming parents' preference to wait. For example, this family's clinician reported, "I said it's up to them...they can get it now or they can wait." Another clinician, describing her response to a reluctant family, said, "I've learned with them to just back off, go on with the rest of the visit, and we'll address it again next year."

Clinicians anticipated resistance from parents regarding the HPV vaccine, particularly because of its perceived
Table 1 Characteristics of study participants

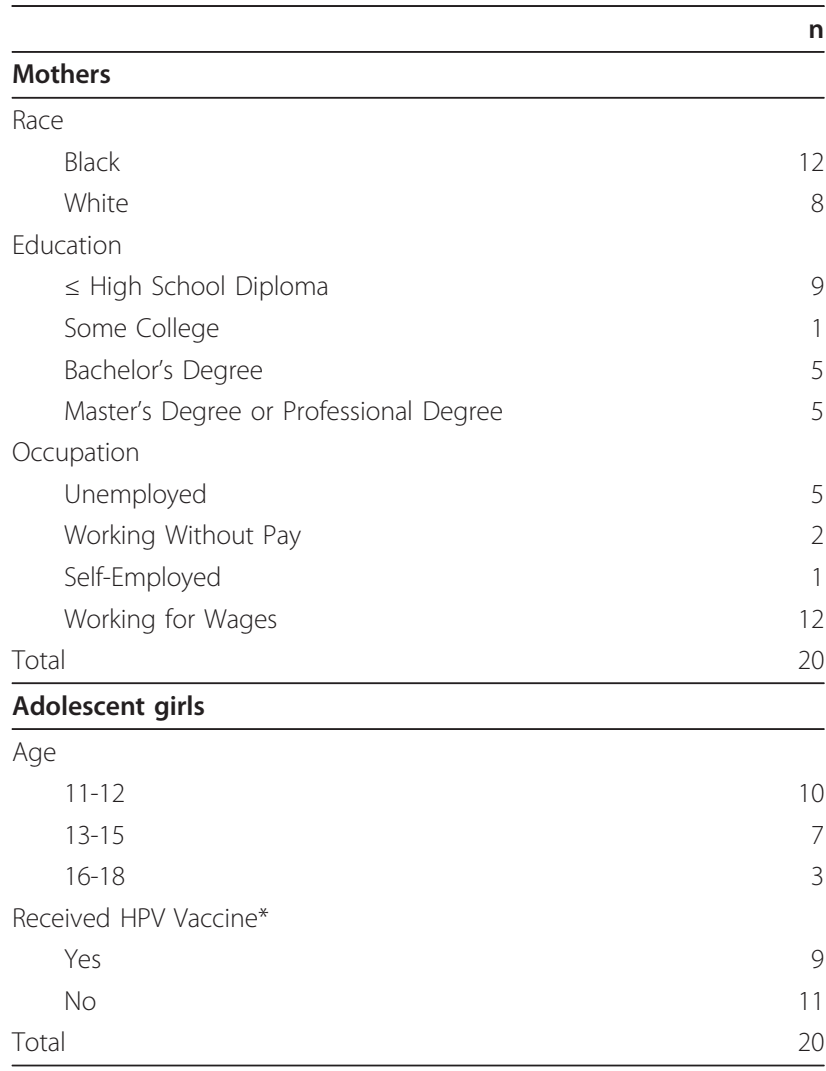

\begin{tabular}{lr}
\hline Clinicians & \\
\hline Race & 2 \\
Black & 15 \\
White & 3 \\
Other & \\
Gender & 16 \\
$\quad$ Female & 4 \\
$\quad$ Male & \\
Practice Setting & 10 \\
Urban Resident Teaching Practice & 10 \\
Other Practices $\wedge$ & \\
Years in Practice (Post-Training) & 3 \\
$\quad<10$ years & 10 \\
10 - 20 years & 7 \\
$>$ 20 years & 20 \\
Total &
\end{tabular}

* Insurance covered HPV vaccine for all subjects.

$\wedge$ Practices located in either an urban or suburban setting with primarily privately.

insured patients and no residents.

association with sexual activity. One clinician whose patient declined the HPV vaccine at the visit noted, "HPV has so many other implications for parents...it's one they fight you on...because you're suggesting that their child is or will be sexually active soon, and they don't want to hear that." Most parents in our sample did associate the need for HPV vaccine with current sexual 
Table 2 Themes and representative verbatim comments

Theme Comment

1. Parents delayed, rather than refused vaccination, and when they expressed reluctance, clinicians were hesitant to engage them in discussion.

\section{Reasons for delay: children not at risk}

"I don't think she need [HPV vaccine] at this time. I guess it's for girls that's having...sex...and I know my daughter and she's not into that right now."

Mother, age 44

"I think I would wait until she's a little bit older, 15 or 16. I just don't think they need it right now, at 13...it's young."

Mother, age 48

"Hopefully [Name] ain't having no sex...but you never know... Hopefully they won't be [sexually active] until they get good and grown. That's what we want but it might not happen."

Mother, age 51

"I don't think my children are at risk where they need to have it."

Mother, age 47

"[The HPV vaccine] is appropriate for when girls start dating, and things along those lines."

Mother, age 45

"I think there's some parents who think 'It's not my kid that's going to be having sex, so they don't need this at all."'

Clinician, non -teaching practice

"[Name] is a perfect example...of a girl that you're pretty confident that at 14 is not sexually active...so you have a little bit of leeway."

Clinician, non-teaching practice

\section{Reasons for delay: concerns about safety and efficacy}

"I probably just want to know more about it and what the risks are...t's new to me...so I need more education on it."

Mother, age 51

"I'd like to know the side effects of it...how long they've been testing it."

Mother, age 30

"I still want to do a little more research...I've heard some pros and cons about it, and since we do have a longer time-line...that's why we're not doing it today."

Mother, age 43

"[Parents] had concerns about how long the vaccine's been out and the safety, and they wanted to have more time to think about it." Clinician, urban teaching practice

Clinician hesitancy

"I don't push it the same way as... [other vaccines]."

Clinician, urban teaching practice

"I will say that I only push it so far...if I had a two-month old who is refusing...IPV or something like that, I might talk to them...I might take up much more time trying to convince them to do that, because I think that the nature of that illness is such that, you know there are devastating consequences...Now, HPV, the truth is that a significant portion of young women will get HPV, but a very large percentage of them will also clear HPV and not actually go on to have cervical cancer. So it's one of those vaccines where I think it's a good idea, but...I don't really twist their arm or anything."

Clinician, urban teaching practice

"She said she'd prefer to wait and get it later...Did she give any specific concerns about why she wants to wait ...No, and I didn't push her on that." Clinician, non-teaching practice

"We probably don't press it as strong as we do with the other [teen vaccines]."

Clinician, non-teaching practice

"I'm comfortable with them waiting on the HPV vaccine; I'm not comfortable with them waiting on DTaP or meningitis."

Clinician, non-teaching practice

2. Clinicians used one of two distinct strategies to present the HPV vaccine. Neither elicited negative responses from families.

Presenting HPV in the same was as other routine vaccines

"I mention it at 11 because that's when the other booster vaccines are due so that helps me in my endeavor to try to vaccinate, by saying you're due for these vaccines."

Clinician, urban teaching practice

"I told her about the vaccines she was due for [including HPV]."

Clinician, urban teaching practice

\section{Presenting HPV as optional and highlighting the risks and benefits of the vaccine}

"He said [HPV] was something that was optional or we could talk about it later, and the others... were not optional." Mother, age 39

"TThe doctor] discussed the side effects....and reducing the chances of her getting the virus...that was helpful." Mother, age 30

"I do explain that it is optional because...it's not like you have to get it for school or anything like that."

Clinician, urban teaching practice

"Part of [why we don't press the vaccine] is, at least currently, [because of the] school regulations... the parents will get messages about [the other vaccines] but they won't get messages about [HPV]."

Clinician, non-teaching practice 
Table 2 Themes and representative verbatim comments (Continued)

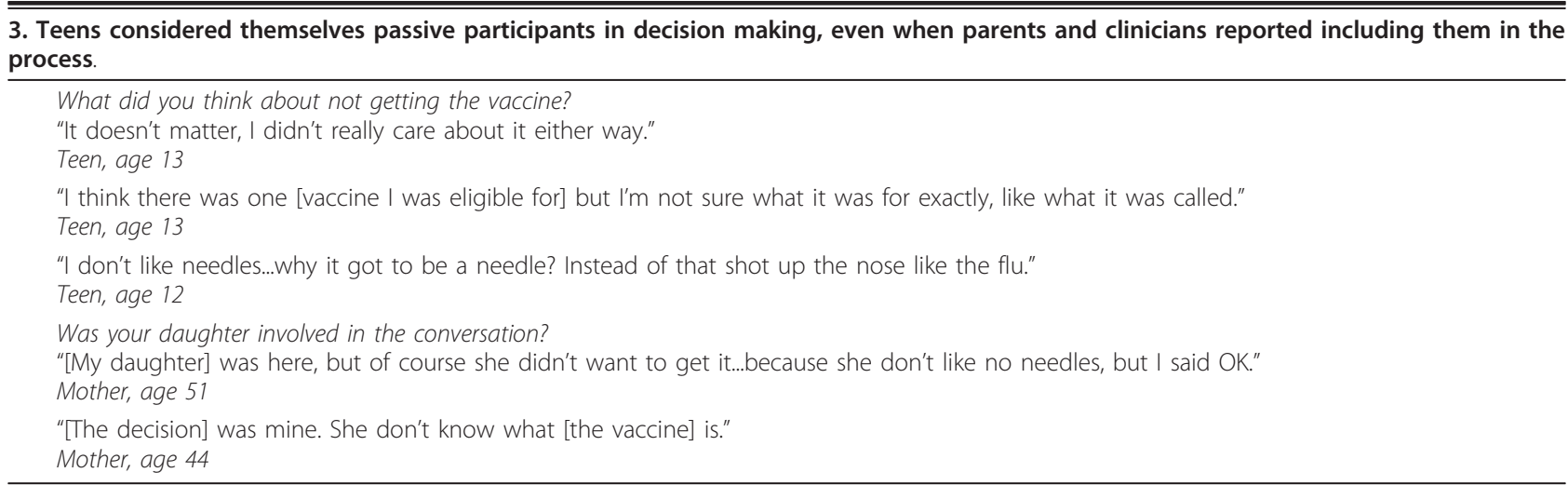

activity. For example, one mother explained, "I don't think I'm ready for her to get [the HPV vaccine]...because she's a good kid and I know she ain't out there sexually... I'm just not worried about that right now." Clinicians expressed difficulty in communicating to parents why the vaccine was recommended to girls who were not yet sexually active. One clinician, after discussing the vaccine with a mother who declined the vaccine because she felt her daughter was not at risk, said, "I think people really misunderstand how ineffective the vaccine is if you give it after they start having sex... [We should think about] how to talk to families about that."

Some clinicians shared parents' view that certain teens were not at risk, and could therefore delay vaccination, which may have influenced their decision not to pursue further discussion. This perception was often based on the characteristics of a child or family's demographic characteristics, such as socio-economic status. One clinician whose patient had delayed the vaccine said, "She hasn't even started puberty yet...it's a very close-knit family, the father's a physician, they live in the suburbs... there's no concern about her being sexually active anytime soon." The mother in this triad recalled, "[The doctor] said [HPV] was something that was optional." Another clinician, whose patient received the vaccine, explained, "The family is a more urban family that has experienced some of the urban realities a little more than some of my suburban families."

Clinicians were also sensitive to parents' worries about the safety and efficacy of the HPV vaccine, especially in the long-term. One clinician explained, "A lot of families have seen advertisements about [the vaccine] and have a lot of concerns." Some parents cited these concerns as a reason for delaying vaccination. One mother said, "Based on the clinical studies I've seen, there's not enough longterm data for immunity for me... I don't believe that the efficacy is there." Her 18 year old daughter explained why she did not receive the vaccine, saying, "I guess my mom...wants to wait until [there is] more research on it."
Another mother added, "I know it's a fairly new vaccine, so I kind of wanted to give it a few years out there...I'm worried about, as these girls get older, if it will affect their reproductive organs."

Some clinicians did not push reluctant families to accept the HPV vaccine because clinicians perceived it was less important than other vaccines. For example, one clinician in a resident teaching setting explained, "Flu would be the example where people very frequently don't want to get it, and I... say I think they're making a very bad decision, that they're putting their child's life at risk...whereas I can't really say that about HPV." The mother in this triad shared this perception, accepting other vaccines at the visit but not HPV.

\section{Clinicians used one of two distinct strategies to present} the HPV vaccine. Neither elicited negative responses from families

Clinicians used two distinct strategies for presenting the HPV vaccine to families. The first was to recommend the HPV vaccine in the same way as other adolescent vaccines, without providing additional details. One clinician in a resident teaching practice whose patient received the vaccine, explained, "I present [HPV] like it's a routine vaccine...I treat it like any other vaccine." Clinicians who used this approach felt that they had more success than those that distinguished the HPV vaccine as unique. In our sample, four of the six patients whose clinicians used this approach received the HPV vaccine at the visit. One clinician said, "Usually the way I present it, they get all the vaccines. If they don't get all the vaccines, it's because they don't want any of them." The mother in this triad accepted the HPV vaccine, and said she was satisfied with the vaccine discussion.

The second approach was to frame the HPV vaccine as "optional" and highlight the risks and benefits of the vaccine. Five of the fourteen teens whose clinicians used this approach received the HPV vaccine at the visit. A clinician described, "With the required vaccines I say these are 
shots she's due for today... and then when I talk about HPV, I say she's also eligible to get the HPV vaccine today, but it's your choice." Another clinician said, "I say it's up to them, that they can get it now or they can wait." Some clinicians who used this strategy focused on parents' concerns about the vaccine being "new." For example, one clinician said, "We spend more time... detailing [why we are recommending it] because it's newer." Others noted that, unlike other routine adolescent vaccines, the HPV vaccine was not required for school attendance, so they presented it separately. Mothers in triads where clinicians used this strategy appreciated the additional information clinicians provided. One mother noted, "When he explained what...[the HPV vaccine] was used for and what it helps prevent...I was comfortable with that."

Clinicians had differing beliefs about how much information they should provide families about the HPV vaccine. Those who used the first approach were aware of the absence of a school mandate, but did not share this with parents. One clinician explained, "I don't really say what's required by school and what's not, and I've never had anyone ask me that." In contrast, a clinician that employed the second strategy said, "It's not a required vaccine so I do educate families that it's a recommended vaccine...I think families have a right to know that as part of their decision-making...I don't feel right in presenting something to a family as non-optional for school when that in fact is not true." In both groups, many clinicians described their approach as consistent across visits. In none of the 20 triads in this sample did parents or adolescents complain about the way the vaccine was presented.

\section{Teens considered themselves passive participants in decision making, even when parents and clinicians reported including them in the process}

Clinician, parent, and adolescent interviews consistently revealed that teens played a minimal role in vaccine decision making. Discussion about vaccines was directed primarily to the mother. Even when clinicians reported engaging both adolescents and parents in discussion about the vaccine, mothers were the primary decision-makers. One 11 year old teen, when asked which vaccines she received, said, "Yes, it's the one that my Mom said but I have no clue what it is. I've never heard of it." Even a 16 year old who was seen alone as part of the visit did not want to discuss the vaccine without her mother present. All teens in our sample reported that they were satisfied with their role in the decision making, and parents were also satisfied with the level of teen involvement. When asked if she would prefer to have participated more in the discussion, one 11 year old who received the vaccine replied, "It doesn't matter. I could talk or not." One mother explained, "She didn't really have a choice, it's my decision as a parent."
The main way that adolescents reported taking part in the vaccine discussion was to express concern about anticipated pain from shots. A 14 year old participant whose mother declined the vaccine noted that she had heard the HPV vaccine was more painful than other vaccines; however, even among participants who had never heard of the HPV vaccine, pain was a primary concern. One 11 year old who received the vaccine, when asked if she would have liked information about vaccines prior to the visit, said, "No, I just don't want to get it. I don't like shots. They hurt." Five teens who expressed concern about pain received the vaccine, and four did not, suggesting that pain may not have impacted vaccine receipt in our sample. While some teens hinted at the connection between maturity and the vaccine, in contrast to their parents, none of the adolescents interviewed mentioned sex in discussing the vaccine (Table 2).

\section{Discussion}

We conducted this qualitative study with mothers, adolescents, and clinicians to better understand discrepancies between research findings that show high HPV vaccine acceptability among parents and adolescents, and high clinician intention to recommend the vaccine, but low rates of receipt. By considering the perspectives within and across each triad interviewed, we identified several themes that may help explain low vaccination rates and inform future interventions.

Our study identified that parents tended to delay, rather than refuse vaccination, and that when parents resist vaccination, clinicians often do not directly address their concerns, contributing to the postponement of vaccination. We found several explanations for this pattern. Confirming results from prior studies, parent concerns about vaccine safety and efficacy were reasons parents chose to delay $[18,26,29]$. However, we found that concerns related to sex were also salient. Parents' perception that teens were not at risk because they were not thought to be currently sexually active often prompted the decision to decline the vaccine and "wait a year" before revisiting the decision. This pattern is concerning since rates of attendance at preventive visits decline after age 11 years and 3 doses are needed for full protection $[9,30]$. These delays may also be prolonged since parents are known to underestimate their children's sexual activity [31,32]. Further research using a longitudinal design would be useful to determine the length of delays and to identify factors that influence when and how vaccine delay turns into vaccine refusal.

Some clinicians, like parents, believed that certain children, especially those in the target age group for vaccination, were not at risk, which may have contributed to their reluctance to encourage vaccination. This perception is concerning since clinicians sometimes reported 
using factors such as urban versus suburban residence and socio-economic status to determine a child's risk. While these characteristics are correlated with sexual initiation on a population level, assessing risk on an individual level using this approach is likely to miss at-risk children and may lead to inequalities if certain groups receive stronger vaccine recommendations [6]. Clinicians also differed in their interpretation of the importance of HPV compared with other vaccines, with some asserting that vaccines such as influenza or the meningitis vaccine justified a stronger recommendation than the HPV vaccine. Finally, some clinicians were uncomfortable engaging families about HPV because it is a sexually transmitted infection (STI) and therefore a sensitive topic for families, a finding that confirms prior qualitative research on HPV vaccine communication [33]. In this context, clinicians were hesitant to "push" for vaccination because of concerns about the impact of this approach on their long-term relationship with families.

As these examples reveal, and as others have found, $[33,34]$ clinicians in our sample identified challenges in communicating with ambivalent families and in presenting the scientific evidence for the HPV vaccine. Effective interpersonal and communication skills as well as knowledge of how to educate families, two areas identified as challenges by clinicians in our study, have been recognized as core competencies by the U.S. Academic Council on Graduate Medical Education (ACGME) and prioritized by policymakers focused on improving healthcare quality $[35,36]$. However, these skills have not historically been emphasized in medical education. Our results highlight the potential benefit of interventions to improve communication between clinicians and parents. Specifically, in the setting of HPV, brief motivational interviews have been suggested as a strategy to engage families who are ambivalent [21]. This technique has been shown to help families identify and clinicians address underlying concerns in a way that allows the clinician to convey respect and empathy while also sharing medical information [37]. Our results suggest that evaluation of this technique in the setting of HPV vaccine decision making is warranted. Additional work is also needed to help clinicians better recognize their own underlying concerns and biases and the ways in which these may impact the presentation and delivery of evidence-based care.

Overall, three prototypes for medical decision making have been defined in the literature: (1) a paternalistic model in which the clinician makes the decision and communicates it to the family, (2) an informed model in which the patient/family gathers information from the clinician and other sources and reaches a decision, and (3) a shared model in which medical and personal information is exchanged and the clinician and family jointly reach a decision [38]. We found that clinicians in our study adopted either the paternalistic or shared model in discussing HPV. Those who used the paternalistic approach believed that they had more success in vaccinating adolescents by presenting HPV as a standard part of the care of early adolescents, without providing additional information about the vaccines. In our sample this appeared to be true, especially among African American patients. However, this qualitative study was not designed to formally test this association. In contrast, those who shared in decision making were less focused on the outcome of vaccine receipt and instead emphasized the importance of involving families in the decision and discussing risks and benefits in detail. Further study is needed to evaluate the impact of both these approaches on decision making, patient satisfaction, and vaccine receipt in diverse primary care populations. Results of this work will help to inform how clinicians should be trained to most effectively address sensitive topics such as HPV vaccination.

Teens in our sample characterized themselves as playing a passive role in vaccine decision making, suggesting that additional strategies for educating and engaging teens about the HPV vaccine as well as other adolescent vaccines may be warranted. Previous studies have found that adolescents are interested in HPV and other STI vaccines; [21] however, this interest may not result in active participation in the primary care setting. Though the American Academy of Pediatrics (AAP) Committee on Bioethics has emphasized the importance of adolescent involvement in decision making,[39] our results suggest that, at least in the short-term, efforts to improve HPV vaccination rates among younger adolescents in the primary care setting may be most effective if targeted primarily at parents. Mothers' approval of vaccination has also been shown to predict vaccine acceptance among older teens,[15] which underscores their central role in decision making.

Teenagers in our study primarily contributed to the vaccine discussion by expressing concerns about pain, a finding supported by other recent qualitative studies $[40,41]$. Although mothers acknowledged their teens' concerns, the conversation with the clinician typically focused on the mother's distinct concerns. Unlike mothers, teenagers in our study did not appear to associate the HPV vaccine with sex. Other studies have shown that some parents worry that vaccination might encourage sexual activity among teenagers; [42] however, our results should help allay these fears. Consistent with findings that teens had limited knowledge about cervical cancer, Papanicolaou smears, [43] and the HPV virus, [40,41] teens in our study, most of whom were 15 or younger, knew very little about what the HPV vaccine prevented. Among those who had heard of the vaccine, none mentioned sexual transmission. Educating teens about sexuality and sexually transmitted infection prevention has not been shown to promote adolescent sexual behavior,[44] a fact that primary care 
pediatricians may find helpful to share with parents specifically concerned about the vaccine's association with sex.

This study had several limitations. We did not observe the visits; therefore, our results are based on participant report. Our sample included mostly younger teens, all of whom were accompanied by a parent; they may be more inclined than older or unaccompanied teens to focus on immediate concerns, such as pain, rather than future benefits. We purposively sampled from pediatric primary care practices within one health system and results may not be generalizable to all settings. As a qualitative study, our aim was to generate themes to enhance our understanding of HPV decision making. Nonetheless, we did assemble a sample of families with diverse racial and educational backgrounds and clinicians working in varied practice settings with a wide range of experience, which mitigates the impact of selection bias. Our finding that teens did not appear to associate the HPV vaccine with sex may have been biased by teens' unwillingness to discuss the issue of sex with the interviewers. Additionally, participants were aware that we were studying adolescent vaccination and this could have potentially biased comments. Still, we elicited a broad and diverse set of responses suggesting that participants openly discussed $\mathrm{HPV}$ vaccine decision making.

\section{Conclusions}

By exploring adolescent, maternal, and clinician perspectives on HPV vaccine decision making at the point of care, we identified several factors that may help explain low rates of receipt. These include parents' tendency to delay vaccination based on beliefs that their teens were not currently at risk for acquiring HPV and clinician reluctance to engage ambivalent parents about their concerns. We also found that teens were passive participants in vaccine decision making, and that clinicians used two distinct approaches in presenting the vaccine to families. In formulating interventions to improve vaccine delivery in primary care, it will be important to develop strategies to promote effective communication between clinicians and parents at the first opportunity, to evaluate approaches such as motivational interviewing to help clinicians engage reluctant parents in discussion, and to measure the impact of distinct clinician decision making styles on HPV vaccine delivery.

\section{Abbreviations}

(HPV): Human papillomavirus; (CHOP): The Children's Hospital of Philadelphia; (PeRC): Pediatric Research Consortium; (STI): Sexually transmitted infection; (ACGME): Academic Council on Graduate Medical Education; (AAP): American Academy of Pediatrics.
}

\section{Acknowledgements}

We thank the network of primary care clinicians, their patients and families for their contribution to clinical research through the Pediatric Research
Consortium (PeRC) at CHOP. In addition, we recognize Richard "Mort" Wasserman for his contribution to protocol development, Jonathan Crossette for his assistance in identifying research subjects, Beth Rezet, Louis M. Bell, and Jim Guevara for helpful comments on this manuscript, and James Massey for his administrative support. This research was supported by an Agency for Healthcare Research and Quality contract

(HHSA29020071001131). In addition, the project described was supported by Award Number K23HD059919 from the Eunice Kennedy Shriver National Institute of Child Health \& Human Development. The content is solely the responsibility of the authors and does not necessarily represent the official views of the Agency for Healthcare Research and Quality or the Eunice Kennedy Shriver National Institute of Child Health \& Human Development or the National Institutes of Health.

\section{Author details}

${ }^{1}$ Center for Pediatric Clinical Effectiveness and Policy Lab, The Children's Hospital of Philadelphia, 3535 Market Suite, Philadelphia, PA, 19104, USA. ${ }^{2}$ Department of Pediatrics, The Children's Hospital of Philadelphia, 3535 Market Suite, Philadelphia, PA, 19104, USA. ${ }^{3}$ Department of Infections Diseases, The Children's Hospital of Philadelphia, 3535 Market Suite, Philadelphia, PA, 19104, USA. ${ }^{4}$ Leonard Davis Institute of Health Economics, The University of Pennsylvania School of Medicine, Colonial Penn Center, 3641 Locust Walk, Philadelphia, PA 19104, USA. ${ }^{5}$ Pediatric Research Consortium, The Children's Hospital of Philadelphia, 3535 Market Suite, Philadelphia, PA, 19104, USA.

\section{Authors' contributions}

$\mathrm{CCH}$ conducted interviews, participated in data analysis and contributed to drafting the manuscript. ALJ conducted interviews and participated in data analysis. KAF helped conceive of the study, participated in data analysis, and contributed to drafting the manuscript. AGF conceived of the study, participated in data analysis, and contributed to drafting the manuscript. All authors read and approved of the final manuscript.

\section{Competing interests}

The authors declare that they have no competing interests.

Received: 18 March 2011 Accepted: 30 August 2011

Published: 30 August 2011

\section{References}

1. US Department of Health and Human Services: Approval Letter - Human Papillomavirus Quadrivalent (Types 6, 11, 16, 18) Vaccine, Recombinant Rockville, MD: Food and Drug Administration; 2006.

2. US Department of Health and Human Services: Approval Letter - Cervarix Rockville, MD: Food and Drug Administration; 2009.

3. Kovacic MB, Castle PE, Herrero R, Schiffman M, Sherman ME, Wacholder S, Rodriguez AC, Hutchinson ML, Bratti MC, Hildesheim A, Morales J, Alfaro M, Burk RD: Relationships of human papillomavirus type, qualitative viral load, and age with cytologic abnormality. Cancer Res 2006, 66:10112-9.

4. Dunne EF, Unger ER, Sternberg M, McQuillian G, Swan DC, Patel SS, Markowitz LE: Prevalence of HPV infection among females in the United States. JAMA 2007, 297:813-9.

5. Winer RL, Feng Q, Hughes JP, O'Reilly S, Kiviat NB, Koutsky LA: Risk of female human papillomavirus acquisition associated with first male sex partner. J Infect Dis 2008, 197:279-82.

6. Eaton DK, Kann L, Kinchen S, Shanklin S, Ross J, Hawkins J, Harris WA Lowry R, McManus T, Chyen D, Lim C, Whittle L, Brener ND, Wechsler H: Youth Risk Behavior Surveillance - United States, 2009. MMWR Surveill Summ 2010, 59:1-142.

7. Paavonen J, Naud P, Salmeron J, Wheeler CM, Chow SN, Apter D, Kitchener H, Castellsague X, Teixeira JC, Skinner SR, Hedrick J, Jaisamrarn U, Limson G, Garland S, Szarewski A, Romanowski B, Aoki FY, Schwarz TF, Poppe WA, Bosch FX, Jenkins D, Hardt K, Wahaf T, Descamps D, Struyf F, Lehtinen M, Dubin G, Greenacre M: Efficacy of human papillomavirus (HPV)-16/18 AS04adjuvanted vaccine against cervical infection and precancer caused by oncogenic HPV types (PATRICIA): final analysis of a double-blind, randomised study in young women. Lancet 2009, 374:301-14.

8. Koutsky LA: Quadrivalent vaccine against human papillomavirus to prevent high-grade cervical lesions. N Engl J Med 2007, 356:1915-27. 
9. Markowitz LE, Dunne EF, Saraiya M, Lawson HW, Chesson H, Unger ER: Quadrivalent human papillomavirus vaccine: recommendations of the Advisory Committee on Immunization Practices (ACIP). MMWR Recomm Rep 2007, 56:1-24.

10. Centers for Disease Control and Prevention: National, state, and local area vaccination coverage among adolescents aged 13-17 years-United States, 2009. MMWR Morb Mortal Wkly Rep 2010, 59: 1018-1023.

11. Widdice LE, Bernstein DI, Leonard AC, Marsolo KA, Kahn JA: Adherence to the HPV vaccine dosing intervals and factors associated with completion of 3 doses. Pediatrics 2011, 127:77-84.

12. Fernandez ME, Allen JD, Mistry R, Kahn JA: Integrating clinical, community, and policy perspectives on human papillomavirus vaccination. Annu Rev Public Health 2010, 31:235-52

13. Brewer NT, Fazekas KI: Predictors of HPV vaccine acceptability: a theoryinformed, systematic review. Prev Med 2007, 45:107-14.

14. Cox DS, Cox AD, Sturm L, Zimet G: Behavioral interventions to increase HPV vaccination acceptability among mothers of young girls. Health Psychol 2010, 29:29-39.

15. Roberts ME, Gerrard M, Reimer R, Gibbons FX: Mother-daughter communication and human papillomavirus vaccine uptake by college students. Pediatrics 2010, 125:982-989.

16. Rosenthal SL, Weiss TW, Zimet GD, Ma L, Good MB, Vichnin MD: Predictors of HPV vaccine uptake among women aged 19-26: importance of a physician's recommendation. Vaccine 2011, 29:890-895.

17. Gerend MA, Weibley E, Bland H: Parental response to human papillomavirus vaccine availability: uptake and intentions. J Adolesc Health 2009, 45:528-31.

18. Dempsey AF, Abraham LM, Dalton V, Ruffin M: Understanding the reasons why mothers do or do not have their adolescent daughters vaccinated against human papillomavirus. Ann Epidemiol 2009, 19:531-8.

19. Conroy K, Rosenthal SL, Zimet GD, Jin Y, Bernstein DI, Glynn S, Kahn JA: Human papillomavirus vaccine uptake, predictors of vaccination, and self-reported barriers to vaccination. J Womens Health 2009, 18:1679-86.

20. Kahn JA, Rosenthal SL, Jin Y, Huang B, Namakydoust A, Zimet GD: Rates of human papillomavirus vaccination, attitudes about vaccination, and human papillomavirus prevalence in young women. Obstet Gynecol 2008, 111:1103-10

21. Zimet GD, Perkins SM, Sturm LA, Perkins SM, Sturm LA, Blair RM, Juliar BE, Mays RM: Predictors of STI vaccine acceptability among parents and their adolescent children. J Adolesc Health 2005, 37:179-86.

22. Feemster KA, Winters SE, Fiks AG, Kinsman S, Kahn JA: Pediatricians' intention to recommend human papillomavirus (HPV) vaccines to 11- to 12-year-old girls postlicensing. J Adolesc Health 2008, 43:408-11.

23. Tissot AM, Zimet GD, Rosenthal SL, Bernstein DI, Wetzel C, Kahn JA: Effective strategies for HPV vaccine delivery: the views of pediatricians. $J$ Adolesc Health 2007, 41:119-25.

24. Kahn JA, Zimet GD, Bernstein DI, Riedesel JM, Lan D, Huang B, Rosenthal SL: Pediatricians' intention to administer human papillomavirus vaccine: the role of practice characteristics, knowledge, and attitudes. J Adolesc Health 2005, 37:502-10.

25. Daley MF, Liddon N, Crane LA, Beaty BL, Barrow J, Babbel C, Markowitz LE, Dunne EF, Stokley S, Dickinson LM, Berman S, Kempe A: National survey of pediatrician knowledge and attitudes regarding human papillomavirus vaccination. Pediatrics 2006, 118:2280-9.

26. Kahn JA, Ding L, Huang B, Zimet GD, Rosenthal SL, Frazier AL: Mothers' intention for their daughters and themselves to receive the human papillomavirus vaccine: a national study of nurses. Pediatrics 2009, 123:1439-45.

27. American Academy of Pediatrics: Recommendations for preventive pediatric health care. [http://practice.aap.org/content.aspx?aid=1599].

28. Kelle U: Different approaches in grounded theory. In The Sage Handbook of Grounded Theory. Edited by: Bryant A, Charmaz K. Los Angeles: Sage Publications; 2007:191-213.

29. Reiter PL, Brewer NT, Gottlieb SL, McRee AL, Smith JS: Parents' health beliefs and HPV vaccination of their adolescent daughters. Soc Sci Med 2009, 69:475-480.

30. Rand CM, Shone LP, Albertin C, Aulinger P, Klein JD, Szilagyi PG: National health care visit patterns of adolescents: implications for delivery of new adolescent vaccines. Arch Pediatr Adolesc Med 2007, 161:252-9.
31. Jaccard J, Dittus PJ, Gordon W: Parent-adolescent congruency in reports of adolescent sexual behavior and in communications about sexual behavior. Child Dev 1998, 69:247-61.

32. O'Donnell L, Stueve A, Duran R, Myint UA, Agronick G, San Doval A, WilsonSimmons R: Parenting practices, parents' underestimation of daughters' risks, and alcohol and sexual behaviors of urban girls. J Adolesc Health 2008, 42:496-502.

33. Brown EC, Little $P$, Leydon GM: Communication challenges of HPV vaccination. Fam Pract 2010, 27:224-229.

34. Krupp K, Marlow LA, Kielmann K, Doddaiah N, Mysore S, Reingold AL, Madhivanan P: Factors associated with intention-to-recommend human papillomavirus vaccination among physicians in Mysore, India. J Adolesc Health 2010, 46:379-384.

35. Levinson W, Lesser C, Epstein R: Developing physician communication skills for patient-centered care. Health Aff 2010, 29:1310-8.

36. Accredidation Council of Graduate Medical Education: 2007 Common program requirements 2010 [http://www.acgme.org/outcome/comp/ compFull.asp], Accessed on July 26

37. Rollnick $\mathrm{S}$, Heather $\mathrm{N}$ : Negotiating behaviour change in medical settings: the development of brief motivational interviewing. J Ment Health 1992, 1:25-38.

38. Charles C, Gafni A, Whelan T: Shared decision-making in the medical encounter: what does it mean? (or it takes at least two to tango). Soc Sci Med 1997, 44:681-92

39. Bartholome WG: Informed consent, parental permission, and assent in pediatric practice. Pediatrics 1995, 96:981-2.

40. Hilton S, Smith E: "I thought cancer was one of those random things. I didn't know cancer could be caught...': Adolescent girls" understandings and experiences of the HPV programme in the UK. Vaccine 2011.

41. Williams K, Forster A, Marlow L, Waller J: Attitudes towards human papillomavirus vaccination: a qualitative study of vaccinated and unvaccinated girls aged 17-18 years. J Fam Plann Reprodu Health Care 2011, 37:22-25

42. Marlow LAV, Waller J, Wardle J: Parental attitudes to pre-pubertal HPV vaccination. Vaccine 2007, 25:1945-52

43. Kahn JA, Chiou V, Allen JD, Goodman E, Perlman SE, Emans SJ: Beliefs about papanicolaou smears and compliance with papanicolaou smear follow-up in adolescents. Arch Pediatr Adolesc Med 1999, 153:1046-54.

44. Kirby D: The impact of schools and school programs upon adolescent sexual behavior. J Sex Res 2002, 39:27-33.

\section{Pre-publication history}

The pre-publication history for this paper can be accessed here: http://www.biomedcentral.com/1471-2431/11/74/prepub

doi:10.1186/1471-2431-11-74

Cite this article as: Hughes et al.: HPV vaccine decision making in pediatric primary care: a semi-structured interview study. BMC Pediatrics 2011 11:74

\section{Submit your next manuscript to BioMed Central and take full advantage of:}

- Convenient online submission

- Thorough peer review

- No space constraints or color figure charges

- Immediate publication on acceptance

- Inclusion in PubMed, CAS, Scopus and Google Scholar

- Research which is freely available for redistribution 